\title{
Polaronic effects in asymmetric quantum wire: An all-coupling variational approach
}

\author{
Phani Murali Krishna ${ }^{\text {a }}$, Soma Mukhopadhyay ${ }^{\text {b,1 }}$, Ashok Chatterjee ${ }^{\text {a,*,1 }}$ \\ ${ }^{a}$ School of Physics, University of Hyderabad, Hyderabad 500046, India \\ ${ }^{\mathrm{b}}$ Shadan Institute of P.G. Studies, 6-2-978, Khairatabad, Hyderabad 500 004, India
}

Received 25 July 2005; received in revised form 9 March 2006; accepted 9 March 2006 by A. Pinczuk

Available online 31 March 2006

\begin{abstract}
An all-coupling variational calculation based on Lee-Low-Pines-Huybrechts (LLPH) theory is performed to study the ground state and the first excited state in an asymmetric polar semiconductor quantum wire that is valid for the entire range of the electron-phonon coupling constant and arbitrary confinement length. It is shown that the polaronic effects are very important and size dependent, if the effective width of the wire is reduced below a certain length scale. It is also shown that asymmetry in a quantum wire can be used as an extra parameter to increase the stability of the polaron. Finally the theory is applied to a realistic CdS quantum wire.
\end{abstract}

(C) 2006 Elsevier Ltd. All rights reserved.

PACS: 71.38.-k; 63.20.Kr; 73.21.Hb

Keywords: A. Asymmetric quantum wire; D. Polaronic effects

\section{Introduction}

Interest in low-dimensional systems has continued unabated for about the last two decades with the advent of more and more sophisticated microfabrication techniques for material growth at the nanoscale. Among the several interesting nanomaterials available today, the one's that have attracted particular attention are zero-dimensional systems or quantum dots [1-4], quantum wires [5], quantum strips [6], quantum discs [7], and quasi-one-dimensional systems like carbon nanotubes $[8,9]$. These systems are interesting both from the point of view of fundamental physics for they provide a unique opportunity in terms of a tiny laboratory to test the important predictions of quantum mechanics and also for their potential applications in opto-electronic devices like quantum dot lasers [10], ultra-fast systems like quantum computers and in microelectronic semiconductor devices like single electron transistors [3].

In the present paper, we shall be interested in a quantum wire. In a quantum wire, the electron's motion is free along

\footnotetext{
* Corresponding author. Tel.: +91 40 23134356; fax: +914023010227.

E-mail address: acsp@uohyd.ernet.in (A. Chatterjee).

${ }^{1}$ Presently at Department of Physics, Bilkent University, Ankara, Turkey.
}

the length of the wire while its motion in the plane normal to the length is confined. Therefore, the energy levels corresponding to electron's motion in the plane normal to the length of the wire are highly quantized and because of this quantization quantum wires are expected to show pronounced quantum effects. Associated with every sharp and discrete energy level however there is a band of quasicontinuous energy levels arising from the free motion of the electron along the length of the wire, which may be referred to as subbands. Since most of the quantum wire structures available today are made of polar semiconductors, one expects that electron-longitudinal-optical (LO) phonon interaction will have pronounced effects on the electronic states of a polar quantum wire and furthermore these effects should be size-dependent and therefore tunable. Quite a few investigations [11,12] have already been made to study the polaronic effects in symmetric quantum wires with different kinds of confining potentials and using different methods. Probably it is worth mentioning at this point that the study of the polaronic effects in quantum dots and wires were inspired by extensive investigations on electron-phonon interactions effects made in the area of quantum wells of polar semiconductors in the eighties (see [13] and references therein). There have also been a host of investigations on several other aspects of quantum well structures (see [14] and references therein). 
Though quite sophisticated and advanced techniques are now available for making quantum dots and quantum wires, fabrication of perfectly symmetric quantum wires is always a difficult problem. Incidentally, however, this difficulty can sometimes appear as a boon in disguise because the inherent asymmetry in the wire can at times be used to our advantage technologically because it offers one more tunable parameter in hand to harvest a lot of new and rich class of physical phenomena. Many authors [15] have studied different effects in asymmetric quantum wires. However, to our knowledge, very few investigations [16] have been made so far on the polaronic effect in asymmetric quantum wires. The purpose of the present paper is to study the polaronic effect in an asymmetric quantum wire for the entire range of the electron-phonon coupling constant and for arbitrary confinement.

\section{The model and formulation}

The hamiltonian for an electron of Bloch mass $m$ moving freely in the $z$-direction with an asymmetric parabolic confinement in the $x$ and $y$ directions with frequencies $\omega_{x}$ and $\omega_{y}$ and interacting with the LO phonons of dispersionless frequency $\omega_{0}$ may be written by modifying the Fröhlich hamiltonian [17] as

$$
\begin{aligned}
H= & -\frac{1}{2}\left(\frac{\partial^{2}}{\partial x^{2}}+\frac{\partial^{2}}{\partial y^{2}}+\frac{\partial^{2}}{\partial z^{2}}\right)+\frac{1}{2}\left(\omega_{x}^{2} x^{2}+\omega_{y}^{2} y^{2}\right) \\
& +\sum_{\vec{q}} b \vec{q}^{\dagger} b \vec{q}+\sum_{\vec{q}}\left(\xi_{q} \mathrm{e}^{-\mathrm{i} \vec{q} \cdot \vec{r}} b \vec{q}^{\dagger}+\text { h.c. }\right)
\end{aligned}
$$

where we have chosen the Feynman units [18] in which $\hbar=$ $m=\omega_{0}=1$. Here, $(x, y, z)$ refers to the electron's position coordinates, $b \vec{q}^{\dagger}(b \vec{q})$ is the creation (annihilation) operator for an LO phonon of wave vector $\vec{q}$ with frequency $\omega_{0}$ and $\left|\xi_{q}\right|^{2}=\left(2 \sqrt{2} \pi / V q^{2}\right) \alpha, \alpha$ being the dimensionless electronphonon coupling constant. We note that the total crystal momentum operator $\vec{P}=-\mathrm{i} \vec{\nabla}+\sum_{\vec{q}} \vec{q} b_{q}^{\dagger} b_{q}$ does not commute with the Hamiltonian (1), though the $z$-component $\vec{P}_{z}=$ $-\mathrm{i}(\partial / \partial z)+\sum_{\vec{q}} q_{z} b_{q}^{\dagger} b_{q}$ does, i.e. $\left[\hat{P}_{z}, H\right]=0 . \hat{P}_{z}$ is thus a conserved quantity. Therefore, one can define an effective mass of the polaron along the $z$-direction, which may be referred to as the longitudinal effective mass.

We seek an all-coupling variational solution of (1) and use a variant of the celebrated Lee-Low-Pines (LLP) transformation method [19], namely the Lee-Low-Pines-Huybrechts (LLPH) technique [20]. Within the framework of the LLPH method we choose the trial wave function as

$$
|\Psi\rangle=\mathrm{e}^{-\mathrm{i} \sum_{\vec{q}}\left(a_{x} q_{x} x+a_{y} q_{y} y+q_{z} q_{z} z\right) b_{\bar{q}}^{\dagger} b_{\bar{q}} \sum \mathrm{e}_{\vec{q}}\left(f_{\bar{q}} b_{\bar{q}}^{\dagger}-f_{\bar{q}}^{*} b_{\bar{q}}\right)}|0\rangle|\Phi(\vec{r})\rangle
$$

where $a_{x}, a_{y}, a_{z}, f_{q}, f_{q}^{*}$ are the variational parameters, $|0\rangle$ is the unperturbed zero-phonon state and $\Phi(\vec{r})$ is the electronic wave function that will be chosen later. The energy is of course given by $E=\langle\Psi|H| \Psi\rangle$. This procedure reduces to the LLP method if we take $a_{x}=a_{y}=1=a_{z}$, which provides a good description in the extended state limit. In the other limit, i.e. in the adiabatic case or the localized state limit, one chooses $a_{x}=a_{y}=0=a_{z}$, which is as expected equivalent to the Landau-Pekar method [17]. By treating $a_{x}, a_{y}$ and $a_{z}$ as variational parameters in the range $(0,1)$, one can thus have a description for the entire coupling parameter space.

For the ground state (GS) we choose the electronic function $\Phi(\vec{r})$ as

$\left|\Phi_{\mathrm{GS}}\right\rangle=\left(\frac{\mu_{x} \mu_{y} \mu_{z}}{\pi^{3 / 2}}\right)^{1 / 2} \mathrm{e}^{-(1 / 2)\left(\mu_{x}^{2} x^{2}+\mu_{y}^{2} y^{2}+\mu_{z}^{2} z^{2}\right)} \mathrm{e}^{-\mathrm{i} p_{0} z}$

where $\mu_{x}, \mu_{y}, \mu_{z}$ and $p_{0}$ are the variational parameters. We now minimize the functional

$\mathbf{J}_{\mathrm{GS}}=\left\langle\Psi_{\mathrm{GS}}|H| \Psi_{\mathrm{GS}}\right\rangle-u_{0}\left\langle\Psi_{\mathrm{GS}}\left|\hat{P}_{z}\right| \Psi_{\mathrm{GS}}\right\rangle$

with respect to the variational parameters $a_{x}, a_{y}, a_{z}, \mu_{x}, \mu_{y}, \mu_{z}, f_{q}$ and $p_{0}$. Here $\left|\Psi_{\mathrm{GS}}\right\rangle$ is given by (2) with $|\Phi\rangle=\left|\Phi_{\mathrm{GS}}\right\rangle$ and $u_{0}$ is the Lagrange multiplier which can be identified as the polaron velocity in the $z$-direction. Minimizing $J_{\mathrm{GS}}$ with respect to $p_{0}$ and $f_{q}\left(\equiv f_{q}^{(0)}\right)$ and expanding the energy in powers of $u_{0}$ we arrive at the expressions for the polaron GS energy and the polaron effective mass as

$$
\begin{aligned}
E_{\mathrm{GS}}= & \frac{\mu_{x}^{2}}{4}+\frac{\mu_{y}^{2}}{4}+\frac{\mu_{z}^{2}}{4}+\frac{\omega_{x}^{2}}{4 \mu_{x}^{2}}+\frac{\omega_{y}^{2}}{4 \mu_{y}^{2}} \\
& -\sum_{\vec{q}} \frac{\left|\xi_{q}\right|^{2} \mathrm{e}^{-\left[\left(\left(1-a_{x}\right)^{2} / 2 \mu_{x}^{2}\right) q_{x}^{2}+\left(\left(1-a_{y}\right)^{2} / 2 \mu_{y}^{2}\right) q_{y}^{2}+\left(\left(1-a_{z}\right)^{2} / 2 \mu_{z}^{2}\right) q_{z}^{2}\right]}}{\left[1+(1 / 2)\left(a_{x}^{2} q_{x}^{2}+q_{y}^{2} q_{y}^{2}+a_{y}^{2} q_{z}^{2}\right)\right]}
\end{aligned}
$$

$m^{*}=1$

$$
+2 \sum_{\vec{q}} \frac{\left|\xi_{q}\right|^{2} q_{z}^{2} \mathrm{e}^{-\left[\left(\left(1-a_{x}\right)^{2} / 2 \mu_{x}^{2}\right) q_{x}^{2}+\left(\left(1-a_{y}\right)^{2} / 2 \mu_{y}^{2}\right) q_{y}^{2}+\left(\left(1-a_{z}\right)^{2} / 2 \mu_{z}^{2}\right) q_{z}^{2}\right]}}{\left[1+(1 / 2)\left(a_{x}^{2} q_{x}^{2}+q_{y}^{2} q_{y}^{2}+a_{y}^{2} q_{z}^{2}\right)\right]^{3}}
$$

Here $a_{x}, a_{y}, a_{z}, \mu_{x}, \mu_{y}, \mu_{z}$, are the six variational parameters that have to be obtained by minimizing $E_{\mathrm{GS}}$ with respect to them.

We take the first excited state electronic wave function as

$\Phi_{\mathrm{ES}}=\left(\frac{2 \mu_{x}^{\prime} \mu_{y}^{\prime} \mu_{z}^{\prime 3}}{\pi^{3 / 2}}\right)^{1 / 2} \mathrm{e}^{-(1 / 2)\left(\mu_{x}^{\prime 2} x^{2}+\mu_{y}^{\prime 2} y^{2}+\mu_{z}^{\prime} z^{2}\right)} z \mathrm{e}^{-\mathrm{i} p_{1} z}$

Let $\left|\Psi_{\mathrm{ES}}\right\rangle$ be the excited state (ES) polaron wave function given by (2) with $|\Phi\rangle=\left|\Phi_{\mathrm{ES}}\right\rangle$. We shall take $p_{1}=p_{0}$ so that the ES wave function is orthogonal to the GS wave function. For the excited state we write $a_{x}=a_{x}^{\prime}, a_{y}=a_{y}^{\prime}, a_{z}=a_{z}^{\prime}$ and $f_{q}=f_{q}^{(1)}$ to distinguish them from the corresponding GS parameters. We now minimize the functional

$\mathbf{J}_{\mathrm{ES}}=\left\langle\Psi_{\mathrm{ES}}\left|\left(H-u_{1} \hat{P}_{z}\right)\right| \Psi_{\mathrm{ES}}\right\rangle$

with respect to $p_{1}$ and $f_{q}^{(1)}$. Here $u_{1}$ is the Langrange multiplier, which as before can be identified as polaron velocity. After some simplifications the ES polaron energy can be written as 


$$
\begin{aligned}
E_{\mathrm{ES}}= & \frac{\mu_{x}^{\prime 2}}{4}+\frac{\mu_{y}^{\prime 2}}{4}+\frac{3 \mu_{z}^{\prime 2}}{4}++\frac{\omega_{x}^{2}}{4 \mu_{x}^{2}}+\frac{\omega_{y}^{2}}{4 \mu_{y}^{2}}-\sum_{\vec{q}}\left(1-\frac{\left(1-a_{z}^{\prime}\right)^{2} q_{z}^{2}}{2 \mu_{z}^{\prime 2}}\right) \\
& \times \frac{\left|\xi_{q}\right|^{2} \mathrm{e}^{-\left[\left(\left(1-a_{x}^{\prime}\right) / 2 \mu_{x}^{\prime 2}\right) q_{x}^{2}+\left(\left(1-a_{y}^{\prime}\right) / 2 \mu_{y}^{\prime 2}\right) q_{y}^{2}+\left(\left(1-a_{z}^{\prime}\right) / 2 \mu_{z}^{\prime 2}\right) q_{z}^{2}\right]}}{\left[1+(1 / 2)\left(a_{x}^{\prime 2} q_{x}^{2}+a_{y}^{\prime 2} q_{y}^{2}+a_{z}^{\prime 2} q_{z}^{2}\right)\right]}
\end{aligned}
$$

where $\mu_{x}^{\prime}, \mu_{y}^{\prime}, \mu_{z}^{\prime}, a_{x}^{\prime}, a_{y}^{\prime}, a_{z}^{\prime}$ are the variational parameters which can be obtained by minimizing $E_{\mathrm{ES}}$ numerically with respect to them.

In the limiting case of symmetric confinement, i.e. for $\omega_{x}=$ $\omega_{y}$, we can take $\mu_{x}=\mu_{y}$ and $a_{x}=a_{y}$ for the GS and $\mu_{x}^{\prime}=\mu_{y}^{\prime}$ and $a_{x}^{\prime}=a_{y}^{\prime}$ for the excited state and we then get back the results for a polaron in a symmetric quantum wire.

\section{Numerical results}

It is customary to define the dimensionless effective confinement lengths in the $x$ and $y$ directions as $l_{x}=1 / \sqrt{\omega_{x}}$ and $l_{y}=1 / \sqrt{\omega_{y}}$. The ground state polaronic correction can then be written as

$\Delta E_{\mathrm{GS}}=E_{\mathrm{GS}}-\frac{1}{2 l_{x}^{2}}-\frac{1}{2 l_{y}^{2}}$

The negative of $\Delta E_{\mathrm{GS}}$ can be called the polaron binding energy, $B E_{\mathrm{GS}}$.

We have taken $l_{y}=0.5$ for all our calculations. In Fig. 1, we have plotted the ground state polaron binding energy as a function of $l_{x}$ for an asymmetric quantum wire for $\alpha=10$. In the same plot we have compared our LLPH results with that of a corresponding symmetric quantum wire and the Landau-Pekar (LP) results of Zhou and Gu [16]. It is clearly evident that our LLPH results are far better than the LP results. Furthermore, so far as $l_{x}$ remains greater than 0.5 , the polaron binding energy for the asymmetric wire continues to be larger than that for the symmetric wire [12]. This is easy to understand because

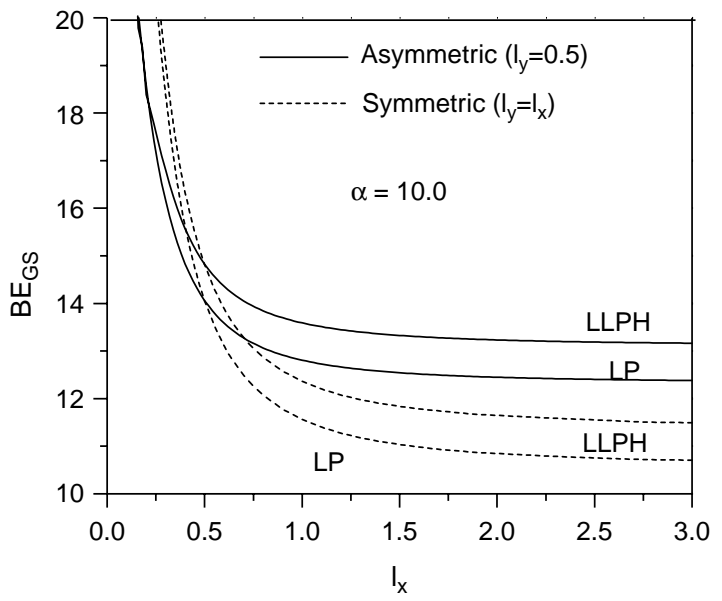

Fig. 1. The GS polaron binding energy (in Feynman units) for an asymmetric quantum wire for $\alpha=10.0$ according to the LLPH method. The results for a corresponding symmetric wire [12] are plotted for the sake of comparison. The $\mathrm{LP}$ results of Zhou and $\mathrm{Gu}[16]$ are also shown for comparison.

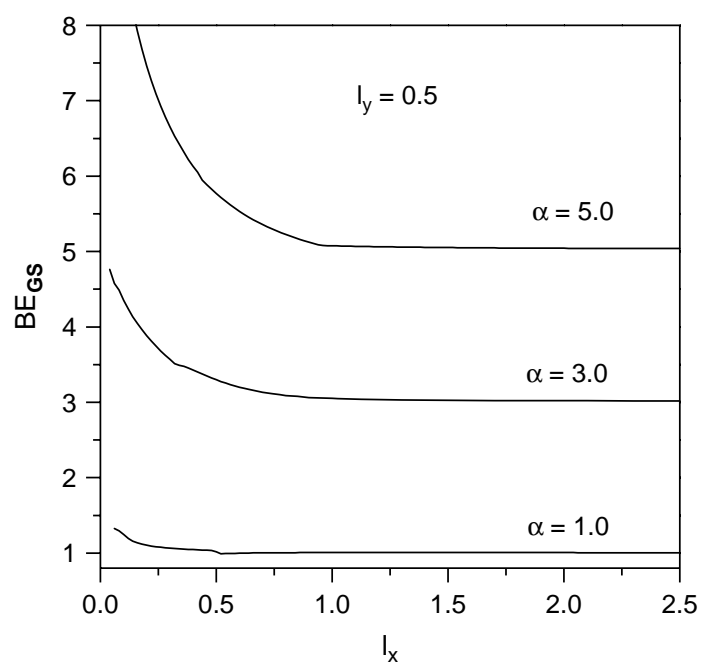

Fig. 2. The GS polaron binding energy (in Feynman units) for an asymmetric quantum wire for $\alpha=1.0,3.0,5.0$.

reduction in the confinement length in any direction increases the polaron binding energy. Of course, the polaronic energy in general increases as the confinements become stronger and stronger.

In Fig. 2, we have shown the GS polaron binding energy for an asymmetric quantum wire for three intermediate coupling values, $\alpha=1,3,5$. However, in this coupling region no other results are available in the literature and therefore we could not make any comparison. In Fig. 3, we have plotted the GS effective mass for arbitrary confinement length $l_{x}$. The effective mass increases very rapidly as we decrease the confinement length below a certain value. Furthermore, the effective mass for the asymmetric wire is larger than the symmetric wire for the range of values we have considered. In Fig. 4, we have plotted the first excited state binding energy as a function of $l_{x}$.

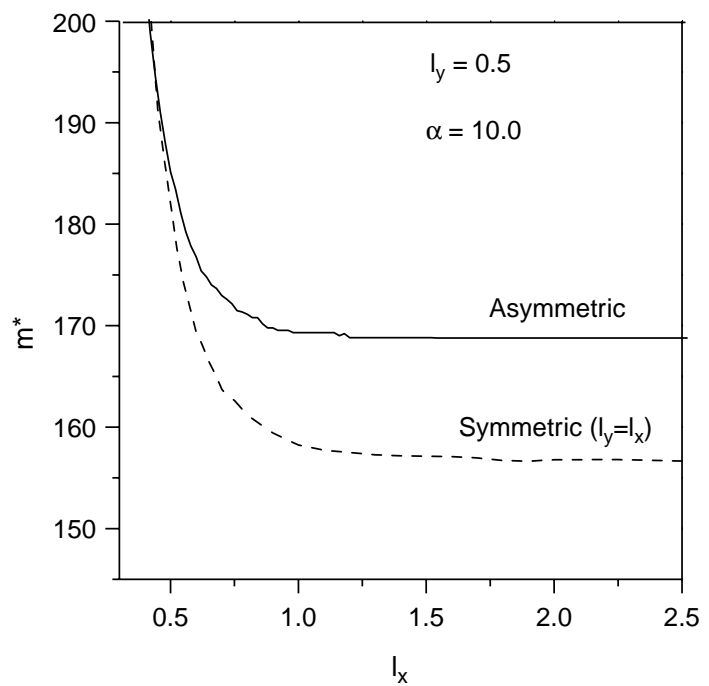

Fig. 3. The effective mass of a polaron in an asymmetric quantum wire for $\alpha=$ 10.0 according to the LLPH method. Results for the corresponding symmetric wire [12] are also shown for comparison. 


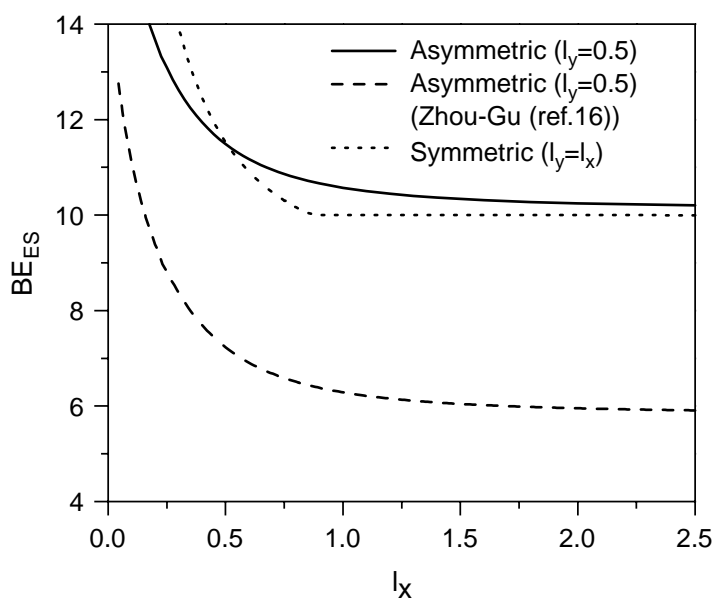

Fig. 4. The ES polaron binding energy (in Feynman units) for an asymmetric quantum wire for $\alpha=10.0$. Results for a corresponding symmetric wire [12] are plotted for the sake of comparison. The LP results of Zhou and Gu [16] are also shown.

One may notice that for $l_{x}>0.5$ the excited state polaron binding energy in the case of an asymmetric quantum wire is larger than that for the symmetric quantum wire [12]. Furthermore, comparison with the LP result of Zhou and Gu [16] shows that the present (LLPH) results are much better.

It would be worthwhile to obtain results for a realistic polar semiconductor asymmetric quantum wire. In Fig. 5, we have, therefore, plotted the GS polaron binding energy for a $\mathrm{CdS}$ quantum wire as a function of the confinement length in real units. We have also compared our result with that of a symmetric CdS quantum wire [12]. One can easily infer from the crossing of the two curves of the figure that the polaron binding energy in an asymmetric quantum wire can be substantially increased if the confinement length in any of the two transverse directions is reduced and this increase in the polaron binding energy should be measurable as it falls in the infrared range for reasonable nanoscale dimensions in the transverse directions.

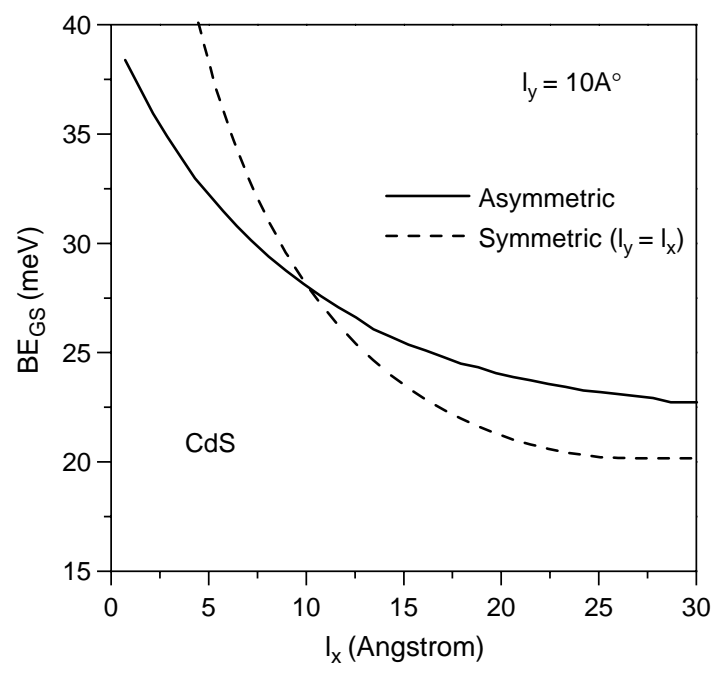

Fig. 5. The polaron binding energy for an asymmetric CdS quantum wire. The results for a symmetric quantum wire [12] are also shown for comparison.

\section{Conclusions}

In conclusion, we have obtained the ground and the first excited state energies and the GS effective mass of a polaron in an asymmetric quantum wire of a polar semiconductor using the all-coupling LLPH variational theory. We have shown that the polaron binding energies can be enhanced substantially by reducing the confinement length in one or both the transverse directions. In fact, the increase in the polaron binding energies becomes very rapid below a certain width of the wire. This effect is even more prominent in the case of the polaron effective mass. To verify the efficacy of our method we have compared our energy results with the Landau-Pekar results of Zhou and $\mathrm{Gu}$ and have shown that our results are much more stable. We have also applied our results to the realistic case of CdS quantum wire and have shown that even by reducing the dimension in one of the transverse directions one can achieve pronounced enhancement in the polaron binding energy in this system, which is measurable.

\section{Acknowledgements}

PMK would like to thank the UPE (University with Potential for Excellence) programme of the University of Hyderabad, India and the CSIR (Council for Scientific and Industrial Research) India for the financial support.

\section{References}

[1] (a) U. Woggon, Optical Properties of Semiconductor Quantum Dots, Springer, Berlin, 1997;

(b) L. Jack, P. Hawrylak, A. Wojs, Quantum Dots, Springer, Berlin, 1998;

(c) D. Bimberg, M. Grundmann, N.N. Ledentsov, Quantum Dot Heterostuctures, Wiley, New York, 1998.

[2] (a) N.F. Johnson, J. Phys.: Condens. Matter 7 (1995) 965;

(b) M.A. Kastner, Phys. Today 46 (1993) 24;

(c) R.C. Ashoori, Nature 379 (1996) 413.

[3] M.A. Kastner, Rev. Mod. Phys. 64 (1992) 849.

[4] S. Mukhopadhyay, A. Chatterjee, Acta. Physica Polonica B 32 (2001) 473 (and references therein).

[5] (a) J. Lee, H.N. Spector, J. Appl. Phys. 54 (1983) 3921;

(b) J. Lee, H.N. Spector, J. Appl. Phys. 57 (1985) 366;

(c) M.H. Degani, O. Hipolito, Phys. Rev. B 35 (1987) 9345;

(d) J. Lee, H.N. Spector, J. Appl. Phys. 97 (2005) 043511.

[6] P.M. Krishna, A. Chatterjee, Physica E 30 (2005) 64.

[7] (a) W. Xie, Physica B 279 (2000) 253;

(b) W. Xie, Phys. Lett. A 252 (1999) 58.

[8] S.J. Tans, M.H. Devoret, H. Dal, A. Thess, R.E. Smalley, L.J. Geerllgs, C. Dekker, Nature 386 (1997) 474.

[9] R. Saito, G. Dresselhaus, Physical Properties of Carbon Nanotubes, Imperial College Press, London, 1998.

[10] (a) P. Bhattacharya, et al., IEEE Trans Electron Devices 46 (1999) 871

(b) N.N. Ledentsov, et al., Semicond. Sci. Technol. 19 (2004) 1183;

(c) R.C. Iotti, F. Rossi, Phys. Rev. Lett. 87 (2001) 146603;

(d) G. Scalari, et al., Phys. Rev. Lett. 93 (2004) 237403.

[11] (a) M.H. Degani, O. Hipólito, Solid State Commun. 65 (1988) 1185;

(b) T. Yildirim, A. Ercelebi, J. Phys.: Condens. Matter 3 (1991) 1271;

(c) T. Yildirim, A. Ercelebi, J. Phys.: Condens. Matter 3 (1991) 4357 
(d) A. Ercelebi, R.T. Senger, Soild State Commun. 97 (1996) 509;

(e) C. Quinghu, R. Yuhang, T. Li, Y. Yabin, Z. Jiao, J. Phys.: Condens. Matter 11 (1999) 4189;

(f) F. Buonocore, G. Iadonisi, D. Ninno, F. Ventriglia, Phys. Rev. B 65 (2002) 205415;

(g) H.J. Xie, Physica E 22 (2004) 906;

(h) G.Q. Hai, F.M. Peeters, J.T. Devreese, L. Wendler, Phys. Rev. B (1993) 12016;

(i) B.S. Kandemir, T. Altanhan, Phys. Rev. B 65 (2002) 054303.

[12] P.M. Krishna, A. Chatterjee, Physica B 358 (2005) 191.

[13] (a) S. Das Sarma, Phys. Rev B 27 (1983) 2590;

(b) S. Das Sarma, Phys. Rev. Lett. 52 (1984) 859;

(c) S. Das Sarma, B.A. Mason, Phys. Rev. B 31 (1985) 5536;

(d) S. Das Sarma, M. Stopa, Phys. Rev. B 36 (1987) 9595;

(e) S. Das Sarma, R. Jalabert, S.R. Eric Yang, Phys. Rev. B 39 (1989) 5516.

[14] (a) A. Pinczuk, S. Schmitt-Rink, G. Danan, J.P. Valladares, L.N. Pfeiffer, K.W. West, Phys. Rev. Lett. 63 (1989) 1633; (b) A.R. Goni, L.N. Pfeiffer, K.W. West, A. Pinczuk, H.U. Baranger, H.L. Stormer, Phys. Rev. Lett. 71 (1993) 4071;

(c) A. Pinczuk, M.G. Lamont, A.C. Gossard, Phys. Rev. Lett. 50 (1986) 2092.

[15] (a) M.R.S. Tavares, G.Q. Hai, G.E. Marques, Phys. Rev. B 68 (2003) 165304;

(b) Y. Fu, et al., Phys. Rev. B 61 (2000) 8306;

(c) Y. Wang, Q. Huag, J. Zhou, Appl. Phys. Lett. 74 (1999) 1412.

[16] H.Y. Zhou, S.W. Gu, Solid State Commun. 91 (1994) 725.

[17] (a) H. Fröhlich, Philos. Mag. 3 (Suppl.) (1954) 325;

(b) T.K. Mitra, A. Chatterjee, S. Mukhopadhyay, Phys. Rep. 153 (1987) 91.

[18] R.P. Feynman, Phys. Rev. 97 (1955) 660.

[19] T.D. Lee, F.E. Low, D. Pines, Phys. Rev. 90 (1953) 297.

[20] (a) W.J. Huybrechts, J. Phys. 10 (1977) 3761;

(b) A. Chatterjee, Ann. Phys. 202 (1990) 320;

(c) S. Mukhopadhyay, A. Chatterjee, J. Phys.: Condens. Matter 11 (1999) 2071. 